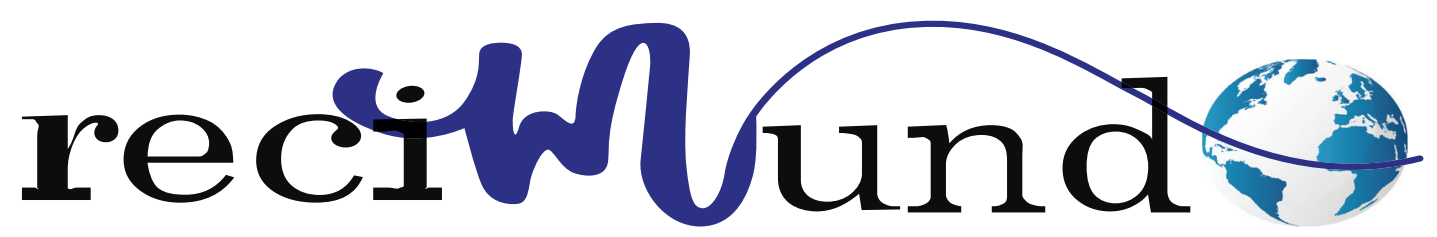

Revista Científica Mundo de la Investigación y el Conocimiento

DOI: 10.26820/recimundo/4.(2).mayo.2020.142-151

URL: http://recimundo.com/index.php/es/article/view/832

EDITORIAL: Saberes del Conocimiento

REVISTA: RECIMUNDO

ISSN: 2588-073X

TIPO DE INVESTIGACióN: Artículo de Revisión

Código UNESCO: 32 Ciencias Médicas; 3201 Ciencias Clínicas

PAGINAS: $142-151$

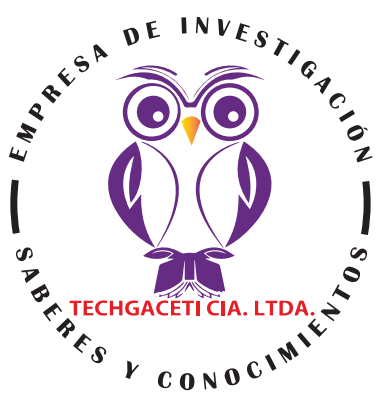

\title{
Complicaciones post operatorias en niños intervenidos por fractura de cadera
}

Post-operative complications in children operated on for hip fracture

Complicações pós-operatórias em crianças operadas por fratura de quadril

Rosa Elena Ambi Muñoz; Andrea Mercedes Celi Mejía²; Alexandra del Carmen Pérez León³; Cristian Eduardo Guevara Benítez ; Ana Valeria Espinoza Castro5; María Belén Mendoza Macias ${ }^{6}$

\section{RECIBIDO: 15/03/2020 ACEPTADO: 20/03/2020 PUBLICADO: 20/05/2020}

1. Médico General; Médico Residente en el Hospital General José María Velasco Ibarra; Tena-Napo, Ecuador; rambim28@gmail.com; (D) https://orcid.org/0000-0001-8921-9591

2. Médico General; Médico residente Hospital Marco Vinicio Iza; Lago Agrio, Ecuador; andremercelmej@gmail. com; iD https://orcid.org/0000-0001-6973-9290

3. Médico General; Hospital Pediátrico Alfonso Villagómez Román; Riobamba, Ecuador; a.perez.leon@hotmail. com; (iD) https://orcid.org/0000-0001-5493-6240

4. Doctor en Medicina; Médico General en Funciones Hospitalarias; Hospital pediátrico Alfonso Villagómez Román; Riobamba, Ecuador; cridu_guebe@yahoo.com; (iD https://orcid.org/0000-0001-8561-7496

5. Médico Cirujano; Hospital de Especialidades Portoviejo; Portoviejo; Ecuador; valy.0592@hotmail.com; iD https://orcid.org/0000-0003-1208-1410

6. Médico General; Médico General en Funciones Hospitalarias - Área de Ginecología - Hospital Rodríguez Zambrano; Manta, Ecuador; belenmendozamacias@gmail.com; iD https://orcid.org/0000-0003-3080-4053

\section{CORRESPONDENCIA \\ Rosa Elena Ambi Muñoz \\ rambim28@gmail.com}

Tena, Ecuador 


\section{RESUMEN}

Las fracturas, en general, son muy comunes en niños y constituyen la cuarta lesión más común entre menores de seis años. De los 0 a los 16 años sufren, al menos, una fractura el $42 \%$ de los niños y el 27\% de las niñas. La mayor incidencia en niños es alrededor de los 15 años y, en niñas, alrededor de los 12 años. Las localizaciones más frecuentes son: 45,1\% en el radio (dominando en su metáfisis y fisis distal), 18,4\% en el húmero (dominando metáfisis y fisis distal), 15,1\% en la tibia, $13,8 \%$ en la clavícula y $7,6 \%$ en el fémur. Las fracturas que afectan a los cartílagos de crecimiento (fisis) representan el $21,7 \%$ de las lesiones. Las fracturas de cadera y raquis son menos frecuentes. Menos del $1 \%$ de las fracturas pediátricas corresponde a las fracturas de cadera. En comparación con el resto de las fracturas pediátricas, las de cadera presentan mayores complicaciones y evoluciones menos favorables. El propósito fundamental del presente estudio es plasmar las principales complicaciones post operatorias en niños intervenidos por fractura de cadera. El diseño de investigación que se llevó a cabo es de tipo documental o bibliográfico. Las principales complicaciones posteriores a la cirugía por fractura de cadera en niños son la osteonecrosis, el cierre fisario prematuro, la no unión y la condrólisis. Se encontró escasa literatura actualizada relacionada con el tema, quizás por la infrecuencia de la presentación de este tipo de fractura en el área pediátrica, no obstante, dada la importancia de sus complicaciones, resulta fundamental realizar estudios de campo y revisiones bibliográficas actualizadas, a los fines de generar un mejor conocimiento del tema y optimizar el manejo existente.

Palabras clave: Complicaciones, Operación, Niños, Fractura, Cadera.

\section{ABSTRACT}

Fractures, in general, are very common in children and constitute the fourth most common injury among children under the age of six. From 0 to 16 years old, at least $42 \%$ of boys and $27 \%$ of girls suffer a fracture. The highest incidence in boys is around 15 years and, in girls, around 12 years. The most frequent locations are: $45.1 \%$ in the radius (dominating its metaphysis and distal physis), 18.4\% in the humerus (dominating metaphysis and distal physis), $15.1 \%$ in the tibia, $13.8 \%$ in the clavicle and $7.6 \%$ in the femur. Fractures that affect growth cartilage (physis) account for $21.7 \%$ of injuries. Hip and spinal fractures are less frequent. Less than $1 \%$ of pediatric fractures correspond to hip fractures. Compared to the rest of pediatric fractures, those of the hip present greater complications and less favorable evolution. The main purpose of this study is to capture the main post-operative complications in children operated on for hip fractures. The research design that was carried out is documentary or bibliographic. The main complications after hip fracture surgery in children are osteonecrosis, premature physeal closure, nonunion, and chondrolysis. Little updated literature was found related to the subject, perhaps due to the infrequency of the presentation of this type of fracture in the pediatric area, however, given the importance of its complications, it is essential to carry out field studies and updated bibliographic reviews, to the in order to generate a better knowledge of the subject and optimize the existing management.

Keywords: Complications, Operation, Children, Fracture, Hip.

\section{RESUMO}

As fraturas, em geral, são muito comuns em crianças e constituem a quarta lesão mais comum em crianças menores de seis anos. De 0 a 16 anos, pelo menos $42 \%$ dos meninos e $27 \%$ das meninas sofrem uma fratura. A incidência mais alta nos meninos é de cerca de 15 anos e, nas meninas, de 12 anos. As localizações mais frequentes são: 45,1\% no raio (dominando sua metáfise e fise distal), 18,4\% no úmero (metáfise dominante e fise distal), 15,1\% na tíbia, 13,8\% na clavícula e 7,6\% no fêmur. As fraturas que afetam a cartilagem do crescimento (physis) são responsáveis por 21,7\% das lesões. Fraturas de quadril e coluna vertebral são menos frequentes. Menos de 1\% das fraturas pediátricas correspondem a fraturas de quadril. Comparadas ao restante das fraturas pediátricas, as do quadril apresentam maiores complicações e evolução menos favorável. O principal objetivo deste estudo é capturar as principais complicações pós-operatórias em crianças operadas por fraturas de quadril. O desenho da pesquisa realizado é documental ou bibliográfico. As principais complicações após a cirurgia de fratura de quadril em crianças são osteonecrose, fechamento físico prematuro, não união e condrólise. Pouca literatura atualizada foi encontrada relacionada ao assunto, talvez devido à pouca frequência da apresentação desse tipo de fratura na área pediátrica, no entanto, dada a importância de suas complicações, é essencial a realização de estudos de campo e revisões bibliográficas atualizadas, para gerar um melhor conhecimento do assunto e otimizar o gerenciamento existente.

Palavras-chave: Complicações, Operação, Crianças, Fratura, Quadril. 


\section{AMBI MUÑOZ, R., CELI MEJíA, A., PÉREZ LEÓN, A., GUEVARA BENITTEZ, C., ESPINOZA CASTRO, A., \& MEN- DOZA MACIAS, M.}

\section{Introducción}

Los traumatismos constituyen la primera causa de mortalidad entre los niños mayores de 1 año y una importante causa de incapacidad permanente. Dentro de las causas más frecuentes de las fracturas en los niños se encuentran las caídas y los accidentes de tránsito o accidentes deportivos. En el caso de la fractura de cadera se considera como una lesión importante en los niños y adolescentes, que puede presentar complicaciones capaces de poner en peligro la vida del paciente. El riesgo de este tipo de fractura se incrementa con la edad, presentándose con un alto índice en personas mayores, no obstante, en la edad infantil es de gran relevancia, pese a su incidencia.

Estas fracturas pueden ocurrir en la cabeza, el cuello, o el área entre o por debajo de los trocánteres (prominencias) del fémur. Como se mencionó anteriormente, las fracturas de cadera son más frecuentes en ancianos, por lo general con osteoporosis y es frecuente que se presenten como resultado de caídas desde el nivel del propio suelo. (Campagne, Fracturas de cadera, 2017)

En materia pediátrica, la Academia Americana de Pediatría (2016) refiere que las fracturas, en general, son muy comunes en niños y constituyen la cuarta lesión más común entre niños menores de seis años. Igualmente indican que "las caídas causan la mayoría de las fracturas en este grupo de edad, pero las fracturas de hueso más graves suelen ser resultado de accidentes en auto".

López Olmedo (2019) estadísticamente refiere acerca de las fracturas en niños y adolescentes lo siguiente:

Entre los 0 y los 16 años sufren, al menos, una fractura, el $42 \%$ de los niños y el $27 \%$ de las niñas. La mayor incidencia en niños es alrededor de los 15 años y, en niñas, alrededor de los 12 años. Las Iocalizacio- nes más frecuentes son: $45,1 \%$ en el radio (dominando en su metáfisis y fisis distal), $18,4 \%$ en el húmero (dominando metáfisis y fisis distal), $15,1 \%$ en la tibia, $13,8 \%$ en la clavícula y $7,6 \%$ en el fémur. Las fracturas que afectan a los cartílagos de crecimiento (fisis) representan el 21,7\% de las lesiones. Las fracturas de cadera y raquis son menos frecuentes. (p. 221.e1)

Por otra parte, Sanabria Ávila, Quesada Jiménez, \& Ramírez Rojas (2010) refieren que las fracturas de cadera son la patología traumática poco frecuente en niños, por lo general, menos del $1 \%$ de las fracturas pediátricas corresponde a fracturas de cadera y la mayor parte de ellas son originadas como consecuencia de trauma de alta energía, correspondiendo a la minoría causas por trauma menor o preexistencia de condiciones patológicas. (p. 27)

Carrillo Muñoz (2009) refiere que las fracturas de cadera en comparación con otras lesiones esqueléticas pediátricas, se relacionan con altos índices de complicaciones y evoluciones desfavorables. Entre las complicaciones más comunes destaca: la osteonecrosis y la no unión o consolidación viciosa. "La mala evolución funcional en los niños con fractura de cadera con frecuencia es debida a la severidad de las lesiones asociadas como lesión cerebral postraumática, por lo tanto, no necesariamente son resultado directo de la fractura de cadera". (p. 267)

Por otra parte, Papalia, Torre, Maffulli, \& Denaro (2019) describen que la fractura de cadera en niños y adolescentes se caracteriza, por lo general, por la presencia de "grave dolor e hinchazón, acortamiento de las piernas, deformidad y puede complicarse por lesiones de tejidos blandos, shock y hemorragia interna. Posterior a la lesión, avascular". Mencionan dentro de las principales complicaciones: la necrosis de la cabeza femoral, la no unión y el cierre prematuro de las fosas. (p. 118) 
Con base a lo expuesto, la gestión de la fractura de cadera en niños va a suponer un problema de gran importancia asistencial. Conocer las principales complicaciones de este tipo de fractura es de gran importancia para su adecuado manejo y la mejor evolución del paciente pediátrico. El objetivo de la presente revisión consiste en plasmar las principales complicaciones que se presentan posterior a una cirugía por fractura de cadera en niños.

\section{Materiales y Métodos}

El presente estudio se fundamenta en una revisión documental bibliográfica y la recopilación de información, que se han obtenido de fuentes digitales, específicamente, de investigaciones publicadas por expertos en la materia, páginas relacionadas con la pediatría y la ortopedia y fuentes oficiales del área de la salud pública como la Academia Americana de Pediatría y la Sociedad Ortopédica Pediátrica de Norte América, entre otros.

En tal sentido, el presente estudio se clasifica como de tipo documental bibliográfico por la naturaleza de su fuente. En atención a lo anterior, la revisión documental es definida como "el proceso dinámico que consiste esencialmente en la recogida, clasificación, recuperación y distribución de la información". (A Latorre, D Rincón, J Arnal, 2003).

Para el desarrollo de la revisión se realizó una búsqueda de material bibliográfico digitalizado que permitió el desarrollo del tema de las principales complicaciones posteriores a una cirugía por fractura de cadera en niños. Para lo cual se usaron bases de datos tales como: MedlinePlus, PubMed, SciELO, Manuales MSD, Medigraphic, entre otras. Para la búsqueda se usaron los siguientes descriptores: "fractura de cadera en niños", "tratamiento de fractura de cadera en niños"; "complicaciones post operatorias por fractura de cadera en niños" y "fracturas en niños". De los resultados de los registros bibliográficos, se filtrados según criterios de idioma, los cuales fueron español e inglés, con una correlación temática, relevancia del tema y una actualidad de fecha de publicación en los últimos diez años.

\section{Resultados}

"Las fracturas de cadera pueden ser producto de un trauma mayor o de uno menor" (ver Figura 1). En los ancianos con presencia de osteoporosis una fractura de cadera puede ocurrir con poco trauma, incluso caminando o cayéndose desde el nivel del propio suelo. (Enciclopedia Médica ADAM, 2020)

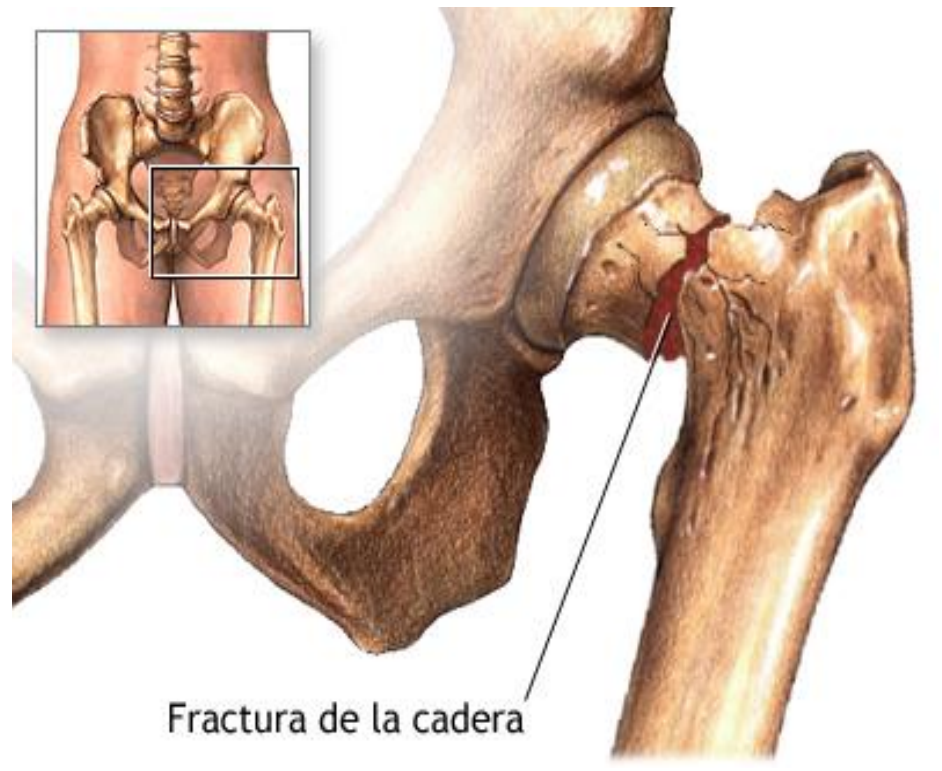

\section{Imagen 1. Fractura de cadera}

Fuente: "Fractura de Cadera". Enciclopedia Médica ADAM. (2020). Recuperado de: https://medlineplus.gov/ spanish/ency/esp_imagepages/18026. $\mathrm{htm}$ 


\section{AMBI MUÑOZ, R., CELI MEJÍA, A., PÉREZ LEÓN, A., GUEVARA BENITEZ, C., ESPINOZA CASTRO, A., \& MEN- DOZA MACIAS, $M$.}

Mientras que, en los niños estas fracturas son menos frecuentes y por lo general se originan por traumas mayores, tales como accidentes de tránsito o deportivos.

Sanabria Ávila, Quesada Jiménez, \& Ramírez Rojas (2010) describen una de las clasificaciones de la fractura de cadera en niños más usada, se trata de la clasificación
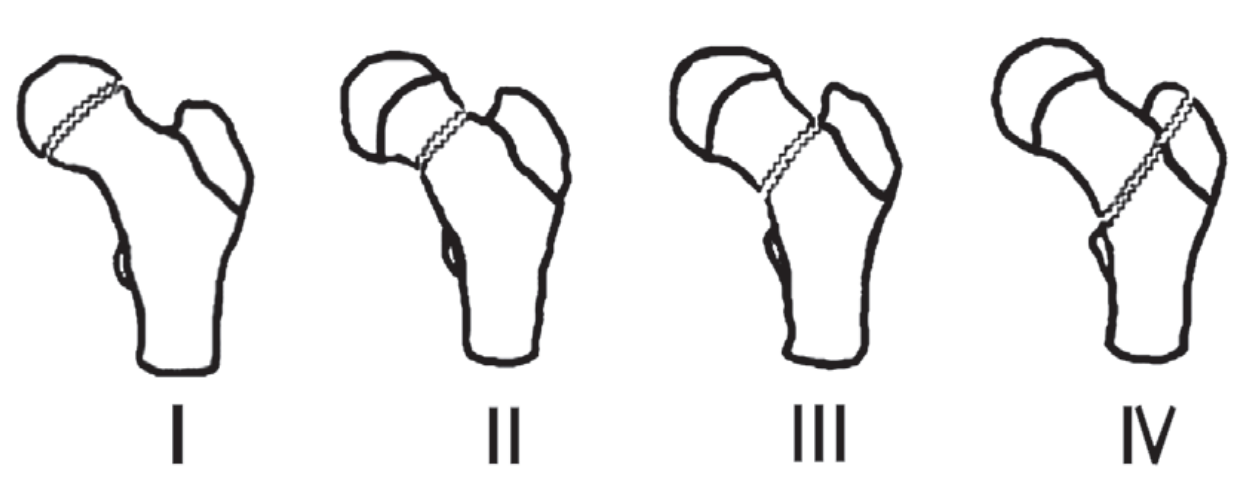

Imagen 2. Clasificación de Delbet para fractura de cadera en edad pediátrica

Fuente: "Fracturas de cadera en edad pediátrica". Sanabria Ávila, G.; Quesada Jiménez, E.; Ramírez Rojas, T. (2010). 67 (591).

El tratamiento de la fractura de cadera es diferente para cada caso y va a depender de algunos factores entre los que se destacan la edad cronológica y la edad ósea, el tamaño del niño y la causa de la fractura.

Algunas fracturas pueden tratarse únicamente con inmovilización en un aparato de yeso. Sin embargo, la mayoría de estas lesiones deben ser tratadas mediante procedimientos quirúrgicos.

Para el tratamiento de las fracturas de cadera en niños, Flores Navarro (2012) sugiere, en líneas generales, que el tratamiento se llevado a cabo en un quirófano con mesa radiolúcida o una mesa de fracturas. Se debe contar con intensificador de imágenes, así como con los tipos de implantes adecuados para la talla del paciente y según se encuentre localizada la fractura. Es preferente llevar a cabo la reducción de manera cerrada y, en el caso de fracturas intracapsulares, se debe considerar la eva-

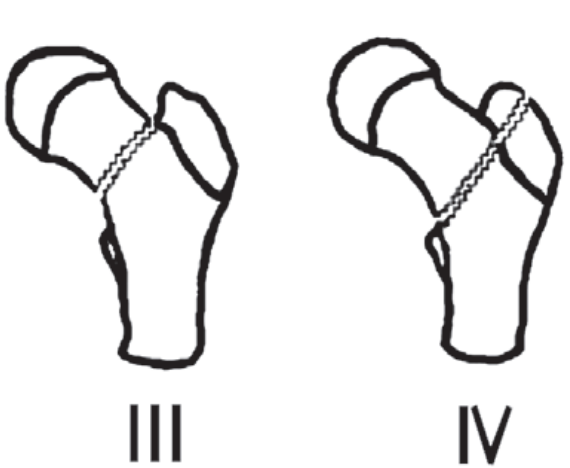

descrita por Delbet (Ver figura 1), el cual las divide de la siguiente manera:

- Tipo I: Transfisiaria (con o sin luxación de la cabeza femoral).

- Tipo II: Transcervical (desplazada o no desplazada)

- Tipo III: Cervico - Intertrocantérica.

- Tipo IV: Intertrocantérica. (p. 29)

cuación del contenido de la articulación por medio de una artrotomía pequeña o punción. No obstante, se recomienda no dudar en proceder con la reducción abierta en caso de ser necesario. Asimismo, agrega las siguientes recomendaciones:

Para todas las fracturas en adolescentes y las tipos II, III y IV que se encuentran desplazadas, a cualquier edad, se recomienda algún medio de fijación interna. Adicionar fijación interna a la inmovilización aumenta considerablemente la estabilidad de la fractura. Es más importante lograr una fijación estable de la fractura que conservar la integridad de la fisis de la cabeza femoral, por lo que es preferible colocar tornillos o clavos a través de la placa fisaria que dejar una fijación con riesgo de ser insuficiente en un intento por no lesionarla. (p. 183, 184)

Las principales complicaciones que pueden presentarse después de la cirugía por fractura de caderas en niños, son las si- 
guientes:

\section{Osteonecrosis}

"En contraste con la mayoría de las fracturas de huesos largos en pediatría, las de cadera presentan una alta incidencia de complicaciones, principalmente osteonecrosis". (Flores Navarro, 2012, p. 180)

Carrillo Muñoz (2009) refiere acerca de la osteonecrosis que esta es la complicación más común que se presenta en las fracturas de cadera en el niño. Acota además en su estudio las siguientes consideraciones acerca de la osteonecrosis:

Recientemente se ha demostrado que el tipo de fractura y la edad en el momento de la lesión son los mejores parámetros para la predicción de osteonecrosis. Las fracturas tipo I, II y III en ese mismo orden, son más susceptibles de presentar osteonecrosis que las fracturas tipo IV. Los niños mayores desarrollan más frecuentemente osteonecrosis debido a la imposibilidad de revascularizar la cabeza femoral y porque en ellos se presentan lesiones tipo I, II y III con más frecuencia que en los niños menores, además, es probable que la necrosis sea resultado de daño vascular en el momento de la fractura. La interrupción del flujo sanguíneo puede ser ocasionada por acodamiento de los vasos a consecuencia del desplazamiento de la fractura o por laceración directa de los vasos sanguíneos por fragmentos fracturarios. Cualquiera de los dos mecanismos puede llevar a ruptura vascular, trombosis e isquemia epifisaria femoral. Otra causa potencial de isquemia es la oclusión de los vasos que irrigan el fémur proximal por aumento de la presión intracapsular secundaria a sangrado en el sitio de la fractura con la cápsula articular íntegra. Con base en estos mecanismos propuestos, la reducción y fijación estable urgente, combinadas con descompresión capsular, pueden reducir la posibilidad de osteonecrosis. Habitualmente la necrosis se diagnostica radiográficamente dentro de los 12 meses posteriores a la lesión, pero puede no ser evidente por algunos años, por lo que se recomienda monitoreo anual hasta la madurez esquelética. (p. 274, 275)

\section{Cierre fisario prematuro}

La Fisis también nombrado como cartílago de crecimiento, placa de crecimiento o placa epifisaria, "es una zona de los huesos largos que se encuentra en la metáfisis. Antes del cierre, la placa de crecimiento es la zona más frágil del hueso y por ello se rompe con frecuencia". (Campagne, 2017)

La Sociedad Ortopédica Pediátrica de Norte América (2010), refiere que dado que la fisis, cartílagos de crecimiento o placas epifisiarias (Ver Figura 3) son la última porción de los huesos en osificarse, por tanto, vulnerables a sufrir fracturas. Los huesos de un niño sueldan más rápido que los huesos de un adulto, lo cual genera dos consecuencias importantes:

- Un niño con una lesión debe ver a un médico lo más rápido posible, para que el hueso reciba el tratamiento adecuado antes de comenzar a soldar. Idealmente, esto significa ver a un especialista ortopédico en un plazo de 5 a 7 días de la lesión, especialmente si se requiere manipulación para alinear el hueso.

- La fractura no necesitará permanecer en un yeso tanto tiempo como requeriría una fractura en un adulto para que suelde.

- Las fracturas del cartílago de crecimiento deben controlarse cuidadosamente para asegurar resultados adecuados a largo plazo. Es por tal razón que las visitas de seguimiento regulares al médico deben continuar por lo menos durante un año después de la fractura. Las fracturas complicadas (tipos III, IV y V) y también las fracturas de fémur y tibia pueden necesitar seguimiento hasta que el niño alcance la madurez esquelética.

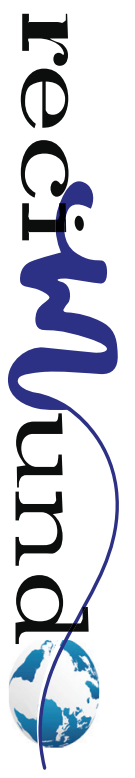




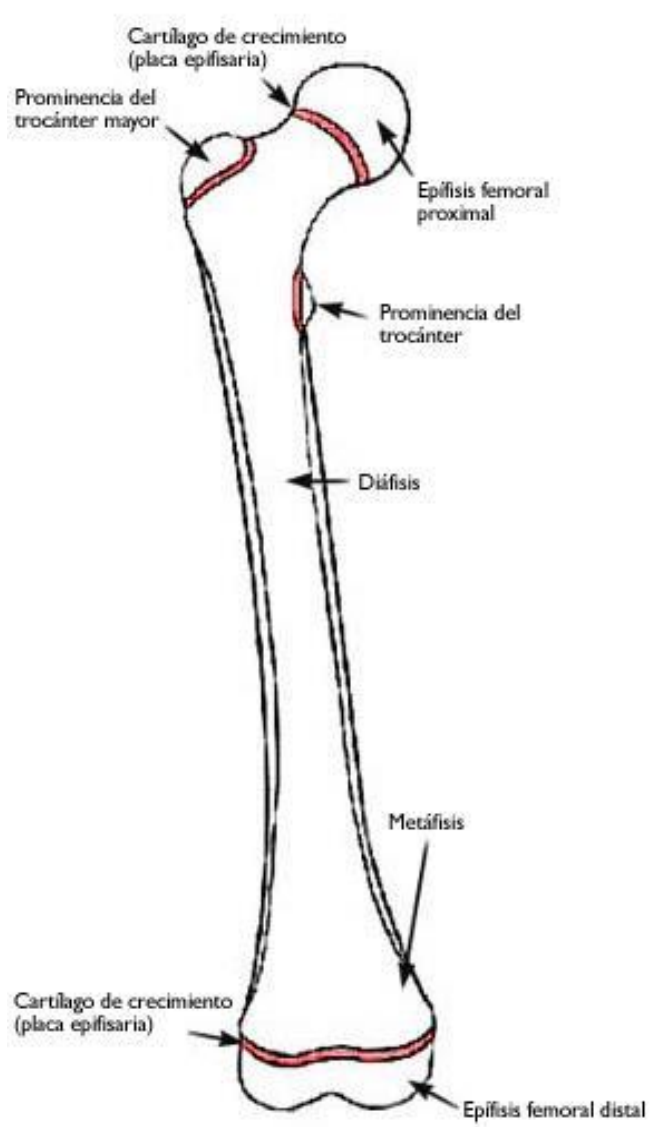

Imagen 3. . Dibujo de un fémur donde se muestra la ubicación de las placas epifisarias en ambos extremos del hueso

Fuente: "Introduction to the Musculoskeletal System". Sullivan, J. A.; Anderson, S. J. (2000), referidos en "Fracturas del Cartílago de Crecimiento. Sociedad Ortopédica Pediátrica de Norte América. (2010)

Los reportes de cierres fisario prematuro varían de un estudio a otro, con un rango de incidencia de 5 al $65 \%$ de los casos, para una diferencia abismal. Algunos de los mecanismos posibles para que resulte esta alteración de crecimiento del fémur proximal pueden ser: trauma directo a la fisis y daño al flujo sanguíneo, los cuales pueden deberse ya sea a la fractura directamente o al tratamiento (cirugía). El cierre fisario prematuro, a su vez, puede ocasionar coxa vara y coxa valga (ángulo cervico-diafisario $>150^{\circ}$ ). La fisis femoral proximal contribuye solo al $15 \%$ de la longitud global de la extremidad o $3 \mathrm{~mm}$ aproximadamente, por año de crecimiento, el cierre fisario completo en niños muy pequeños puede llegar a resultar en una diferencia significativa $(>2 \mathrm{~cm})$ de la longitud de la extremidad. Contrario a lo anterior, casos de fracturas en niños mayores y adolescentes con cierre fisario prematuro desarrollan infrecuentemente una discrepancia en longitud significativa. (Carrillo Muñoz, 2009, p. 275)

Feng Chih, Shu Jui, Jih Yang, \& To (2011) acera del cierre fiseal prematuro reafirman que se ha encontrado una correlación significativa entre la osteonecrosis y el cierre prematuro de las fosas, que se explica dada la interrupción del suministro de sangre del crecimiento placa, la cual puede originarse en el momento de la fractura de cadera o por introducción de un dispositivo interno. (p. 517)

\section{No unión}

No existe un tiempo definido para la solda- 
dura de un hueso, cada uno de ellos y cada región de hueso, poseen su propio tiempo para llegar a la unión ósea. En el caso particular de los niños se produce en tiempos más cortos. Esta patología se le domina "no unión" o "pseudoartrosis". El segundo caso es aplicable con énfasis cuando en la zona de falta de unión, se forma una especie de falsa articulación con membrana y liquido sinovial. Esta no unión ocurre cuando habiendo transcurrido el tiempo suficiente, la fractura no se une. Puede deberse a múltiples causas, por factores anatómicos, propios del traumatismo y mecánicos, no obstante, una de las causas más frecuentes es una mala inmovilización, en donde el medio usado para inmovilizar permite la presencia de macro movimientos continuos en la zona donde se presentó la fractura. Es frecuente que sean los propios pacientes, dada su inquietud y continuo movimiento, los cuales desde el principio del tratamiento incumplen con el cuidado de guardar un reposo adecuado de la zona fracturada, lo cual somete a esfuerzos la zona fracturada con excesivos movimientos, en muchos casos ocasionando rupturas del implante. (Ver figura 4). (Aybar M., 2013)
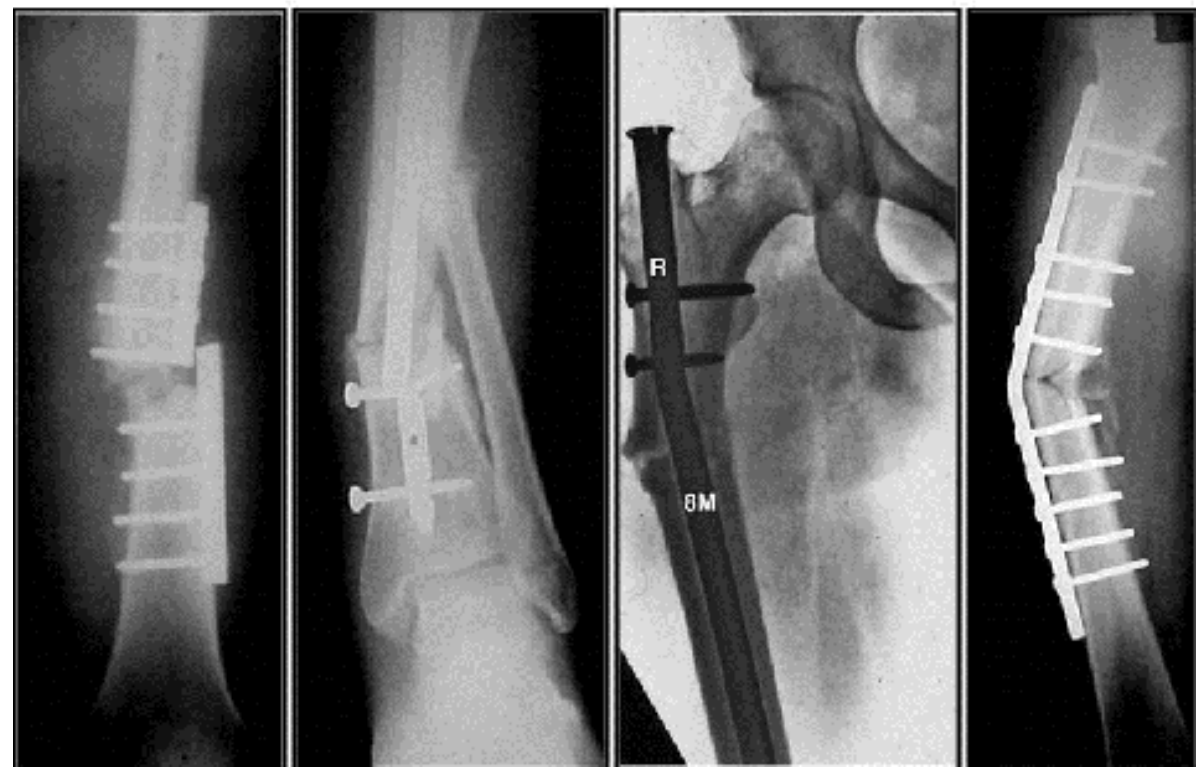

Imagen 4. . Ejemplos de no unión por ruptura del implante

Fuente: “¿Por qué no curan algunas fracturas?". Aybar M., A. (2013).

La no unión también es definida como una falla en la cicatrización de la fractura que se presenta aún después de 4 a 6 meses de tratamiento. En cuanto a datos estadísticos se ha reportado en 1.6 a $10 \%$ de los casos. Las fracturas tipo II y III que son tratadas con fijación fallida o inadecuada, poseen mayores probabilidades de presentar no unión. Entre las otras causas de no unión se puede presentar la infección oculta en la zona de la fractura y osteonecrosis severa del extremo proximal del fémur. En estos casos la mejor opción de tratamiento está representada por una osteotomía val- guizante subtrocantérica. (Carrillo Muñoz, 2009, p.276)

\section{Condrólisis}

La condrólisis se define como la "desaparición del cartílago articular como resultado de la lisis o degeneración del mismo. Se presenta con mayor frecuencia en la articulación coxofemoral y está acompañado de dolor y rigidez". (Clínica Universidad de Navarra, s.f.)

Una de las complicaciones menos frecuen-

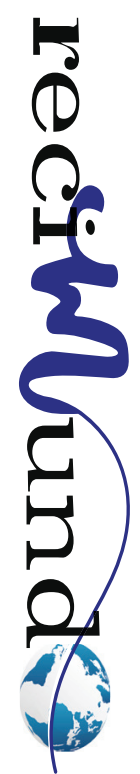




\section{AMBI MUÑOZ, R., CELI MEJÍA, A., PÉREZ LEÓN, A., GUEVARA BENITTEZ, C., ESPINOZA CASTRO, A., \& MEN- DOZA MACIAS, $M$.}

tes es la condrólisis de la cabeza femoral, se observa primero en asociación con osteonecrosis y la restricción de la movilidad de la cadera, coxalgia, y disminución radiográfica del espacio articular son sus principales manifestaciones. La causa probable de esta patología es una deficiencia en la circulación del cartílago articular de la cabeza femoral. "La persistencia de implantes penetrantes en la cabeza femoral también han sido asociados con el desarrollo de condrólisis". (Carrillo Muñoz, 2009, p. 276)

\section{Conclusiones}

Las fracturas en general son lesiones frecuentes en niños, no obstante, la fractura de cadera es una lesión es infrecuente en niños. A pesar de no presentar una alta incidencia, este tipo de fracturas en niños se encuentran asociadas con serias complicaciones, que ponen en riesgo la funcionalidad de la zona afectada y en algunos casos puede llegar a ser mortal.

El tratamiento de estas fracturas va a depender de algunos factores como la edad del niño, la zona específica de la fractura, la causa, entre otras. Algunas de las fracturas de cadera en niños pueden ser tratadas sin necesidad de cirugía, sin embargo, la gran mayoría requiere de este procedimiento.

Las principales complicaciones posteriores a la cirugía por fractura de cadera en niños son la osteonecrosis, la cual es ocasionada por la vulnerabilidad del flujo sanguíneo arterial terminal y dada la anatomía ósea del fémur proximal en estos pacientes. Otras complicaciones más comunes son el cierre fisario prematuro, la no unión y la condrólisis.

Se encontró escasa literatura actualizada relacionada con el tema de las complicaciones post operatorias en niños intervenidos por fractura de cadera, quizás por la infrecuencia de su presentación, no obstante, dada la importancia de sus compli- caciones, es importante realizar estudios de campo y revisiones bibliográficas a los fines de generar un mejor conocimiento del tema y mejorar el manejo existente.

\section{Bibliografía}

A Latorre, D Rincón, J Arnal. (2003). Bases Metodológicas de la Investigación Educativa. Barcelona: Ediciones Experiencia.

Academia Americana de Pediatría. (13 de Abril de 2016). HealthyChildren. Recuperado el 15 de Mayo de 2020, de https://www.healthychildren. org/Spanish/health-issues/injuries-emergencies/ Paginas/Children-And-Broken-Bones.aspx

Aybar M., A. (29 de Abril de 2013). ¿Por qué no curan algunas fracturas? Intramed. Recuperado el 03 de Mayo de 2020, de https://www.intramed.net/ contenidover.asp?contenidoid $=79988$

Campagne, D. (Diciembre de 2017). Manuales MSD. Recuperado el 10 de Mayo de 2020, de https://www.msdmanuals.com/es-mx/hogar/traumatismos-y-envenenamientos/fracturas/fracturas-de-cadera

Campagne, D. (Agosto de 2017). Manueles MSD. Recuperado el 11 de Mayo de 2020, de https:// www.msdmanuals.com/es/professional/lesiones-y-envenenamientos/fracturas/fracturas-pedi\%C3\%A1tricas-de-la-fisis-cart\%C3\%ADIago-de-crecimiento

Carrillo Muñoz, H. (2009). Fracturas de cadera en el niño. Medigraphic, 5(3), 266-278. Recuperado el 12 de Mayo de 2020, de https://www.medigraphic. com/pdfs/orthotips/ot-2009/ot093h.pdf

Clínica Universidad de Navarra. (s.f.). Clínica Universidad de Navara. Recuperado el 11 de Mayo de 2020, de https://www.cun.es/diccionario-medico/ terminos/condrolisis

Enciclopedia Médica ADAM. (10 de Mayo de 2020). MedlinePlus. Recuperado el 21 de Enero de 2020, de https://medlineplus.gov/spanish/ency/esp_ imagepages/18026.htm

Feng Chih, K., Shu Jui, K., Jih Yang, K., \& To, W. (21 de Febrero de 2011). Complicaciones de fractura de cadera en niños. Chang Gung Med J, 34(5), 512519. Recuperado el 15 de Mayo de 2020, de https://www.researchgate.net/publication/51755264

Flores Navarro, H. H. (2012). Fractura de caderas en niños y adolescentes. Medigraphic, 8(3), 179-186. Recuperado el 12 de Mayo de 2020, de https:// www.medigraphic.com/pdfs/orthotips/ot-2012/ 
ot123i.pdf

López Olmedo, J. (2019). Fracturas infantiles más frecuentes. Esguinces y epifisiolisis. Pediatría Integral, 23(4), 221.e1 - 221.e14. Recuperado el 11 de Mayo de 2020, de https://www.pediatriaintegral.es/wp-content/uploads/2019/xxiii04/06/n4221e1-14_JorgeLopez.pdf

Papalia, R., Torre, G., Maffulli, N., \& Denaro, V. (07 de Febrero de 2019). Fractura de cadera en niños y adolescentes. British Medical Bulletin, 129, 117128. doi:10.1093/bmb/ldz004
Sanabria Ávila, G., Quesada Jiménez, E., \& Ramírez Rojas, T. (2010). Fracturas de cadera en edad pediátrica. Revista Médica de Costa Rica y Centroamérica, 67(591), 27-36. Recuperado el 10 de Mayo de 2020, de https://www.medigraphic.com/ pdfs/revmedcoscen/rmc-2010/rmc10591g.pdf

Sociedad Ortopédica Pediátrica de Norte América. (Enero de 2010). Orthoinfo. (A. A. Ortopédicos, Productor) Recuperado el 10 de Mayo de 2020, de https://orthoinfo.aaos.org/es/diseases--conditions/fracturas-del-cartilago-de-crecimiento-growth-plate-fractures

\section{CITAR ESTE ARTICULO:}

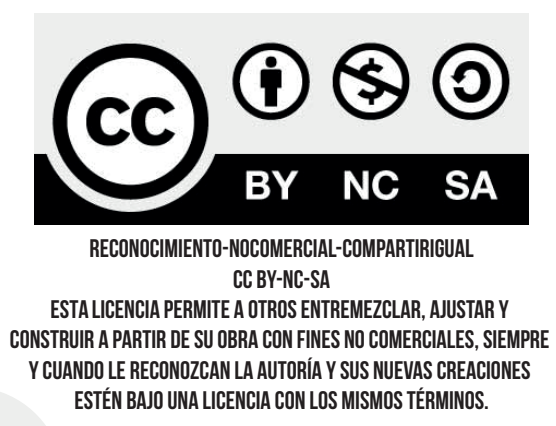

Ambi Muñoz, R., Celi Mejía, A., Pérez León, A., Guevara Benítez, C., Espinoza

Castro, A., \& Mendoza Macias, M. (2020). Complicaciones post operatorias en niños intervenidos por fractura de cadera. RECIMUNDO, 4(2), 138-147. doi:0.26820/recimundo/4.(2).mayo.2020.142-151 